\title{
Nonstationary Robust Control for Vibration of Elevator Rope*
}

\author{
Masatsugu OTSUKI ${ }^{* *}$, Kazuo YOSHIDA***, Toshiaki NAKAGAWA ${ }^{\dagger}$, \\ Hiroyuki KIMURA ${ }^{\dagger \dagger}$ and Shigeru FUJIMOTO ${ }^{\dagger \dagger}$ \\ ** Department of System Design Engineering, Keio University \\ 3-14-1 Hiyoshi, Kohoku-ku, Yokohama, Kanagawa, 223-8522, Japan
}

\author{
Present Affiliation: Institute of Space and Astronautical Science, Japan Aerospace Exploration Agency \\ E-mail: peke@2000.jukuin.keio.ac.jp \\ *** Department of System Design Engineering, Keio University \\ 3-14-1 Hiyoshi, Kohoku-ku, Yokohama, Kanagawa, 223-8522, Japan \\ $\dagger$ Development Department, Toshiba Elevator and Building Systems Corporation \\ 1 Toshiba-cho, Fuchu-shi, Tokyo, 183-8511, Japan \\ $\dagger$ Power and Industrial Systems R\&D Center, Toshiba Corporation \\ 1 Toshiba-cho, Fuchu-shi, Tokyo, 183-8511, Japan \\ t† Power and Industrial Systems R\&D Center, Toshiba Corporation \\ 4-1, Ukishima-cho, Kawasaki-ku, Kawasaki, Kanagawa, 210-0862, Japan \\ Present Affiliation: Department of Mechanical Design Engineering, Shonan Institute of Technology
}

\begin{abstract}
It is performed to suppress the transverse vibration of the elevator ropes on a high-rise building caused by resonance between building-sway and rope-sway. The elevator rope has the characteristics due to its flexibility and length-varying and some constraints for the active vibration control. Hence, for compensating the influence of the timevarying characteristics and spillover, the nonstationary robust controller which has the robustness against unstructured and structured uncertainties is employed for the active vibration suppression of the rope. The suppression and robust stabilizing performances of the controller are demonstrated through the numerical calculations for the case in which the building is subjected to the ground disturbance like an earthquake and the tension of the rope varies. Thereupon, the control input system actuated by an alternate current motor is located right below the traction sheave and then it moves the rope directly in the presence of gaps between the rope and actuator. Consequently, the nonstationary robust controller shows the advantages in terms of the robustness on the uncertainties and the performance as compared to the optimal controller; and then it can adequately suppress the transverse vibration of the rope.
\end{abstract}

Key words : Elevator Rope, Time-Varying System, Vibration Control, Nonstationary Robust Control, Flexible Structure

\section{Introduction}

In recent years, with increasing height of buildings, moving distance in vertical direction and velocity in terms of an elevator have been brought up; in particular, the ultra speed elevator, which has the maximum speed approximately 1000 meters per minute, has installed in the skyscraper Taipei Financial Center in 2005. The high speed and long distance elevator is generally moved by using main ropes attached to an elevator cage and a traction sheave; and then the main ropes have more flexible feature and lower natural frequency than those of normal ropes. Thus, transverse vibration of the rope is caused by resonance with building-sway induced by a long-term earthquake and strong wind ${ }^{(1)}$. Hence, the suppression of the vibration of the rope is expected in skyscrapers and high-rise buildings, which have a low natural frequency. Currently, as the countermeasures against this rope-sway problem, the elevator op- 
eration has been taken in such a manner that depends on the magnitude of the building-sway or the passive dampers are located in the hoistway of the elevator to prevent increase of the rope-sway ${ }^{(2)}$. They are needed to shorten recovery duration for the elevator in case in which the vibration of the rope occurs due to small-to-medium-sized earthquakes and to protect the entire elevator system from big earthquakes. This paper presents an active control method to solve the above rope-sway problem, which is expected from the view of social requirements of increasing efficiency of the elevator operation and reduction of maintenance time of the elevator system. The active vibration suppression of the main ropes is particularly focused in this paper and it is implemented by using a control device and sensors located in the vicinity of the upper end of the main rope. The location of the actuator and sensors is limited into both ends of the rope or their vicinity owing to length-varying of the rope.

Furthermore, the elevator rope is a type of a time-varying system due to change in its length and natural frequency with time; hence, the vibration control by a conventional stationary method is ineffective in terms of stabilization of the system. There are some studies on the robust control for a linear time-varying system. The synthesis method based on the game theory was proposed for the output feedback controller ${ }^{(3)}$ and the synthesis of the controller which can simultaneously implement the position and vibration control for the flexible structure was also unified by using the time-varying weightings ${ }^{(5)}$. With regard to controlling the length-varying wire, Takagi et al. ${ }^{(4)}$ proposed the gain-scheduled control method based on a linear matrix inequality for the vibration control of a cable in a tower crane but the change in the length of the cable alone was considered. In addition, the authors performed the optimal control of the transverse vibration of the elevator rope caused by resonance due to building-sway through the numerical calculation ${ }^{(6)}$. The time-varying controller generally can be categorized into a gain scheduled controller or nonstationary one and the gain scheduled controller is then enabled the time-varying control by switching stationary controllers on the basis of the parameter variation; meanwhile, the nonstationary controller designed on the basis of the priori information on the variation of the controlled object implements a smooth continuous control. The common characteristics of the above controllers are to be designed by using the synthesis method with high flexibility and to increase the order of the controller as compared to a time-invariant one. Consequently, there is few applications for the actual system $^{(7),(8)}$. Further, the robustness of the control system is influenced by uncertainties owing to the flexibility of the elevator rope, fluctuation for the tension of the ropes, variation of the tension due to incoming and outgoing passengers and employment of the reduced-order model as a synthesis model. Therefore, it is required that the controller is synthesized with the high robustness against the uncertainties due to parameter error and variation.

In this paper, the vibration control using the control device which moves the elevator rope directly is proposed as a solution of the rope-sway problem in a high-rise building; it is purpose to establish the practical and robust method of the vibration suppression of the elevator rope without the influence of the nonlinearity and uncertainty of the control device. In order to verify the feasibility of the proposed method and the performance of the designed controller, the numerical calculation which simulates the suppression of the transverse vibration of the elevator rope is performed. In Ref. (8), the vibration of the elevator rope was reduced by moving the traction sheave, which is located at the top boundary of the main rope. However, there are some practical constraints: need of a high power actuator and big margin of system safety; therefore, the method to control the rope directly is adopted in this paper. From the viewpoint of the reduction of contacting of the rope with the control device and extension of the life cycle of the rope, a gap is then added into the position between the control device and rope. First, the nonlinearity of the gap in the control device is examined and the dynamics of the rope and control device with an alternate current motor are also verified. Second, the nonstationary robust control considering the uncertainties, which was proposed in Ref. (9), is applied to the vibration control of the elevator rope in this paper and the practical design method of the robust controller is considered with the verification of its robust stabilizing 

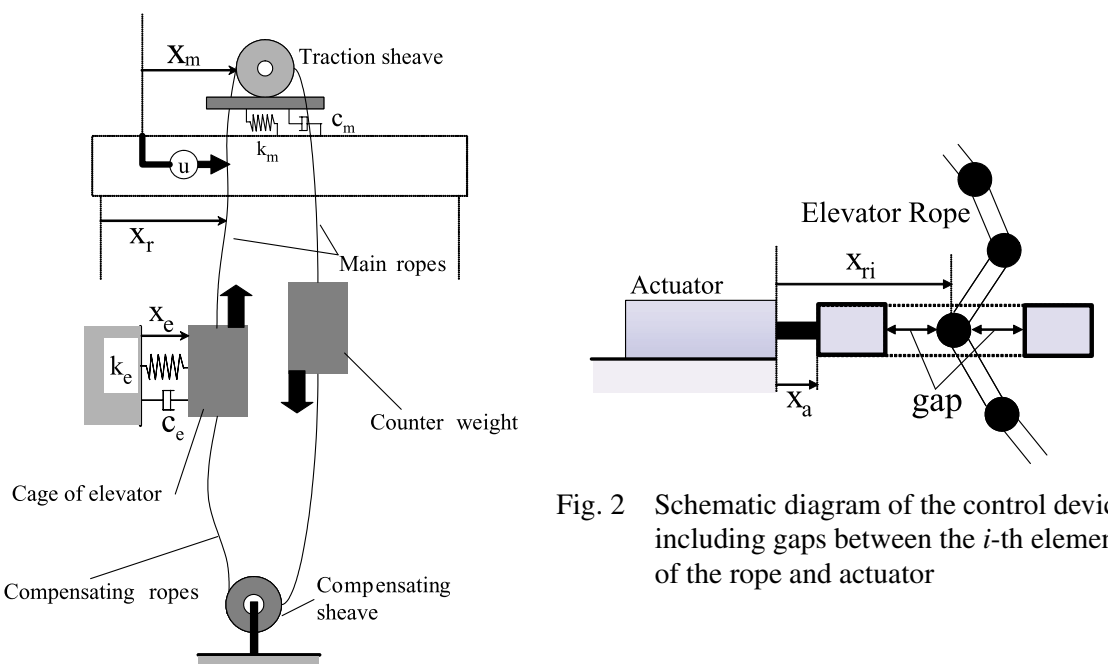

Fig. 2 Schematic diagram of the control device including gaps between the $i$-th element of the rope and actuator

Fig. 1 Schematic diagram of the controlled object

performance.

Finally, in order to downsize the control device and to reduce the number of the sensors, the feasibility of the simultaneous vibration control for the multiple elevator ropes using the single control device is verified through the numerical calculation for the case in which the tension of the ropes fluctuates.

\section{Controlled object}

This study deals with the vibration control problem in an elevator system as shown in Fig1 which consists of a traction sheave, a cage, main ropes, a counter weight, compensating ropes, a sheave and a control device with gaps as shown in Fig2. The compensating ropes are employed for regulating the tension of the main ropes; however, the only vibration of the main ropes, which lift up the cage, is focused in this paper. Further, the vibration of the rope will be reduced by moving it directly in the vicinity of its top boundary by using the control device with the gaps between the rope and actuator.

\subsection{Formulation of an elevator rope}

In this section, the equation of motion of the rope will be constructed. It is assumed that the elevator system is situated in an over 500 meters high-rise building and the longitudinal vibration of the rope can be ignored. The transverse vibration of the rope caused by resonance between the building and rope-sway is considerably larger than the longitudinal one. It is also supposed that the behavior of the building, elevator rope, traction sheave and cage are constrained in the horizontal direction; and the traction sheave and cage are fixed at the top story of the building and rail in the hoistway, respectively, through the spring and damper. Although there are the multiple main ropes in the elevator system, the formulation of the single rope is performed in this section. The rope is assumed to be a wire through its formulation. A wave equation ${ }^{(1),(8)}$ of a length-varying wire is expressed by the following equation:

$$
\rho A\left[\frac{\partial}{\partial t}+v(t) \frac{\partial}{\partial s}\right]^{2} u(s, t)-\frac{\partial}{\partial s} T(s, t) \frac{\partial u(s, t)}{\partial s}+c(s, t)\left[\frac{\partial}{\partial t}+v(t) \frac{\partial}{\partial s}\right] u(s, t)=0
$$

where $s$ is the coordinate along the rope; $u(s, t)$ the distributed parameter of the deflection of the rope in the horizontal direction depending on the vertical position; $t$ the arbitrary time; $\rho A$ the line density of the rope; $T(s, t)$ the tension of the rope depending on the vertical position; $v(t)$ the changing velocity in the rope length; and $c(s, t)$ the damping coefficient per unit length of the rope depending on the vertical position. The equation of motion of the rope is constructed by using the FEM method as $u(s, t)=N(s) \boldsymbol{x}_{r}(t)$. And it is then assumed that $N(s)$ is 
the first order shape function; thus, the equation of motion of an element of the rope is derived from the following equation:

$$
\begin{aligned}
& {\left[\begin{array}{cc}
2 \alpha(t) & \alpha(t) \\
\alpha(t) & 2 \alpha(t)
\end{array}\right]\left[\begin{array}{c}
\ddot{x}_{r i}(t)+\ddot{z}_{b i}(t) \\
\ddot{x}_{r i+1}(t)+\ddot{z}_{b i+1}(t)
\end{array}\right]+\left[\begin{array}{cc}
2 \gamma_{r}(t)-d_{c}(t) & \gamma_{r}(t)+d_{c}(t) \\
\gamma_{r}(t)-d_{c}(t) & 2 \gamma_{r}(t)+d_{c}(t)
\end{array}\right]\left[\begin{array}{c}
\dot{x}_{r i}(t) \\
\dot{x}_{r i+1}(t)
\end{array}\right]+} \\
& {\left[\begin{array}{cc}
\beta(t)-d_{k}(t) & -\beta(t)+d_{k}(t) \\
-\beta(t)-d_{k}(t) & \beta(t)+d_{k}(t)
\end{array}\right]\left[\begin{array}{c}
x_{r i}(t) \\
x_{r i+1}(t)
\end{array}\right]=0} \\
& \alpha(t)=\rho A l(t) / 6, l(t)=(l(0)+v(t) t) / n, d_{c}(t)=\rho A v(t), \gamma_{r}(t)=c(s, t) l(t) / 6 \\
& \beta(t)=\left(T(s, t)-\rho A v^{2}(t)\right) / l(t), d_{k}(t)=0.5 c(s, t) v(t)
\end{aligned}
$$

where $x_{r i}(t)$ is the relative displacement of the $i$-th discrete element from the building; $\ddot{z}_{b i}(t)$ the absolute acceleration of the building depending on the vertical position of the $i$-th discrete rope element; $n$ the discrete number in the formulation of the rope; and it is also assumed that the rope does not directly interact with an external force. In addition, $d_{c}(t)$ and $d_{k}(t)$ are the advection terms depending on the length-varying velocity of the rope. In this FEM model, the number of the discrete element of the rope is not changed; however, the length of the rope changes with time. Consequently, the integrated equation of motion of the elevator system is expressed by the following equation on the basis of the superposition of the equations of motion of the $n$ discrete elements of the rope, traction sheave and cage.

$$
\begin{aligned}
& \boldsymbol{M}_{r}(t) \ddot{\boldsymbol{x}}_{d}(t)+\boldsymbol{C}_{r}(t) \dot{\boldsymbol{x}}_{d}(t)+\boldsymbol{K}_{r}(t) \boldsymbol{x}_{d}(t)=\boldsymbol{F}_{u}(t)+\boldsymbol{F}_{e}(s, t) \\
& \boldsymbol{x}_{d}(t)=\left[\begin{array}{lllllll}
x_{m} & x_{r 2} & \cdots & x_{r i} & \cdots & x_{r n} & x_{e}
\end{array}\right]^{T}
\end{aligned}
$$

where $x_{m}=x_{r 1}$ and $x_{e}=x_{r n+1}$. The details of all the matrices except for $F_{u}(t)$ and $F_{e}(s, t)$ are omitted in this paper. The input matrix $\boldsymbol{F}_{u}(t)$ is described by

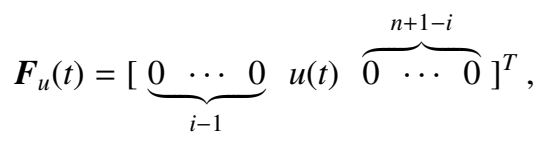

where $u(t)$ is the direct control force into the rope. It is assumed that the elevator system is located in a high-rise building; thus, the vibration of the high-rise building is excited by an earthquake or strong wind; however, the strong wind is daily problem. Since the disturbance into the rope is not due to a direct force, the vibration of the rope is caused by resonance with that of the building; consequently, both external disturbances are available for the verification of the vibration problem. In this paper, the earthquake is adopted because it has the wide frequency components. The disturbance into the rope is derived from the response of the model of the over 500 meters high-rise building, which is constructed on the basis of the shearing model with the five stories. Thus, the disturbances into the discrete elements of the rope are derived from the absolute acceleration of the top story of the building as follows:

$$
\boldsymbol{F}_{e}(s, t)=-\boldsymbol{M}_{r}(t)\left[\ddot{z}_{b 1} \cdots \ddot{z}_{b n+1}\right]^{T}=-\boldsymbol{M}_{r}(t)\left[\boldsymbol{k}_{b}(s, t)\left(\ddot{x}_{b 5}-\ddot{z}_{e}\right)+\ddot{z}_{e}\right]
$$

where $\ddot{z}_{e}$ is the acceleration of the earthquake and $\boldsymbol{k}_{b}(s, t)$ is the coefficient which is derived from dividing the acceleration of the disturbance into each element of the rope on the arbitrary time by the relative acceleration of the top story of the building for the case in which the building is assumed a cantilever whose vibration is expressed by the only first mode.

A noncontact sensor for measuring the relative displacement of the elevator rope from the building and contact sensors for measuring the relative displacements and velocities of the traction sheave and cage, respectively, are considered as the sensors to observe the state of the system. The location of all the sensors and actuator is limited into the vicinity of the top boundary of the rope. Here, the shift of their location arises from the length-varying discrete elements of the rope. Therefore, the lengths of the discrete elements of the rope near the control device are fixed to calculate correctly the control force at the same position, and the lengths of the residual elements are varied due to time. 


\subsection{Control device with gaps}

The active control device is located at 10 meters below the traction sheave and the active vibration control is then performed by moving the rope directly. If the control device touched the rope continuously, the rope is more subject to wear. Hence, gaps are located between the control device and rope; namely, the control input is implemented with the impact between the control device and rope. Thereupon, the control device is formulated for each case in which the rope is contacted with the control device or not. When the rope contacts with the control device, the dynamics of the control device and rope is formulated in a manner that depends on the contacting side, left and right, respectively. Moreover, the impact between the rope and control device is expressed by the coefficients of a spring and damping which are considerably larger than those of the discrete element in the model of the rope. The schematic representation of the control device and gaps are shown in Fig2. Further, for all the cases, the control force $u(t)$ is described by the following equations:

$$
u(t)=\left\{\begin{array}{l}
0 \text { if }\left|x_{r i}-x_{a}\right| \leq g a p \\
-k_{a}\left(x_{r i}(t)-x_{a}(t) \pm g a p\right)-c_{a}\left(\dot{x}_{r i}(t)-\dot{x}_{a}(t)\right) \text { if } \mp\left(x_{r i}-x_{a}\right)>g a p
\end{array}\right.
$$

where $x_{r i}(t)$ is the relative displacement of the $i$-th element of the rope from the building at the located position of the control device; $x_{a}(t)$ the relative displacement of the actuator of the control device; and gap the width of the gap. The parameters $k_{a}$ and $c_{a}$ are arbitrarily identified by minimizing the rope deflection beyond the gap-width and removing the vibration with the high frequency component. An alternate current motor is adopted as the actuator in the control device and its dynamics can be described in the form of the equivalent direct-current motor ${ }^{(10)}$. Therefore, the dynamics of the actuator is expressed by the following differential equation:

$$
\begin{aligned}
& {\left[\begin{array}{c}
\dot{x}_{a}(t) \\
\ddot{x}_{a}(t) \\
\dddot{x}_{a}(t)
\end{array}\right]=\left[\begin{array}{ccc}
0 & 1 & 0 \\
0 & 0 & 1 \\
0 & -\alpha_{a} & -\beta_{a}
\end{array}\right]\left[\begin{array}{c}
x_{a}(t) \\
\dot{x}_{a}(t) \\
\ddot{x}_{a}(t)
\end{array}\right]+\left[\begin{array}{c}
0 \\
0 \\
f_{a}(t)
\end{array}\right]} \\
& f_{a}(t)=\left\{\begin{array}{l}
0 \quad \text { if }\left(\left|x_{r i}-x_{a}\right| \leq \text { gap }\right) \\
\gamma_{a} e(t)+\alpha_{a} \epsilon_{a} u(t)+\epsilon_{a} \dot{u}(t) \text { if }\left(\left|x_{r i}-x_{a}\right|>\text { gap }\right)
\end{array}\right. \\
& \alpha_{a}=\frac{K_{t}^{2} d_{m}}{L I}, \beta_{a}=\frac{R}{L}, \gamma_{a}=\frac{K_{t}}{L I}, \epsilon_{a}=\frac{d_{f}}{I}, d_{m}=\frac{1}{d_{f}}, I=\left(J d_{m}+m_{a} d_{f}\right) \text {, }
\end{aligned}
$$

where $e(t)$ is the direct-current voltage $[\mathrm{V}] ; K_{t}$ the torque constant $[\mathrm{Nm} / \mathrm{A}] ; d_{f}$ the conversion coefficient from torque to force $[\mathrm{m} / \mathrm{rad}] ; L$ the self-inductance $[\mathrm{Vs} / \mathrm{A}] ; J$ the sum of the inertia of the motor axis and so on $\left[\mathrm{kgm}^{2}\right] ; m_{a}$ the mass of the control device $[\mathrm{kg}]$; and $R$ the resistance of the motor $[\Omega]$. The performance of the controller and behavior of the controlled object are evaluated by solving the dynamics of the elevator system and actuator simultaneously. The controller is designed by the combined equation of motion as the state of the motor $x_{a}(t)$ coincides with the state of the element of the rope $x_{r i}$ at the position where the control device is located. The actuator is formulated on the basis of the equation of motion of the equivalent direct-current motor; as the result, a control input of the proposed controller shown in the next section is calculated as a value of voltage. Actually, the velocity command is employed for a signal into the servo control system of the alternate current motor.

\section{Nonstationary robust control method}

\subsection{Formulation of the control problem}

It is assumed that the actual controlled object can be completely described by the following linear time-varying system.

$$
\dot{\boldsymbol{x}}(t)=\boldsymbol{A}(t) \boldsymbol{x}(t)+\boldsymbol{B}(t) u(t)+\boldsymbol{D}(t) \boldsymbol{w}(t)
$$

And the nominal model of the controlled object for the synthesis of a controller is given by the following equation:

$$
\dot{\boldsymbol{x}}_{n}(t)=\boldsymbol{A}_{n}(t) \boldsymbol{x}_{n}(t)+\boldsymbol{B}_{n}(t) u(t)+\boldsymbol{D}_{n}(t) \boldsymbol{w}(t) .
$$


To conform the system described by Eq.(9) to the system in Eq.(8), the complicated formulation, nonlinear function, consideration of higher order modes and/or a FEM model with a large number of node are needed in most instances. Hence, the dimension of Eq.(9) is typically lower than that of Eq.(8). Thus, the difference between Eqs.(8) and (9) indicates the modeling error; consequently, it causes spillover of a controller and observer. Further, the complete realization of the parameter identification for the controlled object is hardly performed; particularly, a nonparametric identification of a time-varying system is complicated. The characteristics of the controlled object may be also changing with passing time. Hence, from the view of the robustness against the uncertainties, it is required to design a controller with considering the uncertainties due to modeling error and parameter variation.

The $\mathrm{H}^{\infty}$ norm of the closed loop transfer function from the supposed worst disturbance $\boldsymbol{w}(t)$ to the performance output $z(t)$ is the criteria in the robust stabilization with the $\mathrm{H}^{\infty}$ control theory ${ }^{(11)}$ and on the basis of the $L_{2}$ induced norm in the time domain the criteria is transformed into the following equation:

$$
\min _{u}\left\|G_{z w}(s)\right\|_{\infty}=\min _{u} \sup _{\boldsymbol{w}} \frac{\|\boldsymbol{z}(t)\|_{2}}{\|\boldsymbol{w}(t)\|_{2}}=\gamma^{*}<\gamma
$$

which is also transformed in the following equation as a criterion function:

$$
J_{\infty}=\min _{u} \max _{\boldsymbol{w}} \int_{0}^{\infty} \boldsymbol{z}^{T}(t) \boldsymbol{z}(t)-\gamma^{2} \boldsymbol{w}^{T}(t) \boldsymbol{w}(t) d t<0
$$

where the criteria in Eq.(10) is the basis at the arbitrary time. However, it is valid that the sensitivity parameter $\gamma$ is the time-varying function because the controlled object is a timevarying system and the uncertainty and disturbance, which influence on the controlled object, are also nonstationary. Hence, it is assumed that the value of $\gamma$ changes with passing time; thus, it is transformed as $\gamma \rightarrow \gamma(t)$. Equation (11) is redesigned as follows.

$$
J=\min _{u} \max _{\boldsymbol{w}} \int_{0}^{t_{f}} \boldsymbol{z}^{T}(t) \boldsymbol{z}(t)-\gamma^{2}(t) \boldsymbol{w}^{T}(t) \boldsymbol{w}(t) d t
$$

where to finish the control in the finite time $t_{f}$ is supposed. In this study, the sensitivity function $\gamma(t)$ is previously calculated and it is employed for the synthesis of the controller. However, the controller should be synthesized on the basis of the known time-varying parameter or nonstationary feature of the controlled object.

\subsection{Nonstationary robust controller}

In this section, the control problem is formulated with considering the uncertainties so as to satisfy the criterion function in Eq.(12). First of all, the state equation (9) of the controlled object is reconstructed with inclusion of the structured and unstructured uncertainties and time-varying weightings; as the result, the generalized plant is obtained by the followings.

$$
\begin{aligned}
\dot{\boldsymbol{x}}_{z}(t) & =\boldsymbol{A}_{z}(t) \boldsymbol{x}_{z}(t)+\boldsymbol{B}_{1}(t) \boldsymbol{w}_{d}(t)+\boldsymbol{B}_{2}(t) u(t) \\
\boldsymbol{z}_{h}(t) & =\boldsymbol{C}_{1}(t) \boldsymbol{x}_{z}(t) \quad+\boldsymbol{D}_{12}(t) u(t) \\
\boldsymbol{y}(t) & =\boldsymbol{C}_{2}(t) \boldsymbol{x}_{z}(t)+\boldsymbol{D}_{21}(t) \boldsymbol{w}_{d}(t)
\end{aligned}
$$

where it is assumed that $\boldsymbol{D}_{11}(t)=0$ and $\boldsymbol{D}_{22}(t)=0$ because the generality of the control problem does not disappear. The details of the construction of the augmented system considering the uncertainties are referred to Ref. (9). The criterion function against the above generalized plant is described on the basis of Eq.(12) as follows:

$$
\begin{aligned}
& J\left(u^{o}, \boldsymbol{w}_{d}^{o}\right)=\min _{u} \max _{\boldsymbol{w}_{d}} \int_{0}^{t_{f}}\left[\boldsymbol{z}_{h}^{T}(t) \boldsymbol{z}_{h}(t)-\gamma^{2}(t) \boldsymbol{w}_{d}^{T}(t) \boldsymbol{w}_{d}(t)\right] d t \\
& =\min _{u} \max _{\boldsymbol{w}_{d}} \int_{0}^{t_{f}}\left[\boldsymbol{x}_{z}^{T}(t) \tilde{\boldsymbol{Q}}(t) \boldsymbol{x}_{z}(t)+2 \boldsymbol{x}_{z}^{T}(t) \tilde{\boldsymbol{S}}(t) u(t)+u^{T}(t) \tilde{\boldsymbol{R}}(t) u(t)-\gamma^{2}(t) \boldsymbol{w}_{d}^{T}(t) \boldsymbol{w}_{d}(t)\right] d t \\
& \tilde{\boldsymbol{Q}}(t)=\boldsymbol{C}_{1}^{T}(t) \boldsymbol{C}_{1}(t), \tilde{\boldsymbol{S}}(t)=\boldsymbol{C}_{1}^{T}(t) \boldsymbol{D}_{12}(t), \tilde{\boldsymbol{R}}(t)=\boldsymbol{D}_{12}^{T}(t) \boldsymbol{D}_{12}(t)
\end{aligned}
$$


where $u^{o}$ is the optimal control input and $\boldsymbol{w}_{d}^{o}$ is the supposed worst disturbance. They are the solutions of this min-max problem but $\boldsymbol{w}_{d}^{o}$ is the solution of maximization problem:

$$
J\left(u, \boldsymbol{w}_{d}^{o}\right)=\max _{\boldsymbol{w}_{d}} J\left(u, \boldsymbol{w}_{d}\right),
$$

and it is described by the equation:

$$
\boldsymbol{w}_{d}^{o}(t)=\gamma^{-2}(t) \boldsymbol{B}_{1}(t) \boldsymbol{X}_{w}(t) \boldsymbol{x}_{z}(t) .
$$

where $\boldsymbol{X}_{w}(t)$ is the positive definite symmetric matrix as a solution of the Riccati differential equation derived from Eq.(17). Thereupon, $u^{o}$ is the solution of the minimization problem:

$$
J\left(u^{o}, \boldsymbol{w}_{d}\right)=\min _{u} J\left(u, \boldsymbol{w}_{d}\right),
$$

and it is the optimal control input given by the equation:

$$
u^{o}(t)=-\tilde{\boldsymbol{R}}^{-1}(t)\left(\boldsymbol{B}_{2}^{T}(t) \boldsymbol{X}_{u}(t)+\tilde{\boldsymbol{S}}(t)\right) \boldsymbol{x}_{z}(t) .
$$

where $\boldsymbol{X}_{u}(t)$ is also the positive definite symmetric matrix as a solution of the Riccati differential equation derived from Eq.(19). From the above consideration, the problem shown in Eq.(16) is converted into the problem to obtain the saddle point solution by implementing the optimal control input $u^{o}$ in the presence of the worst disturbance $\boldsymbol{w}^{o}$. The solution can satisfy the following inequality on the basis of Eqs.(17) and (19).

$$
J\left(u^{o}, \boldsymbol{w}_{d}\right) \leq J\left(u^{o}, \boldsymbol{w}_{d}^{o}\right) \leq J\left(u, \boldsymbol{w}_{d}^{o}\right),
$$

and it is implemented with the following output feedback controller in the state space descrip$\operatorname{tion}^{(3)}$.

$$
\begin{aligned}
\dot{\hat{\boldsymbol{x}}}(t) & =\hat{\boldsymbol{A}}(t) \hat{\boldsymbol{x}}(t)+\hat{\boldsymbol{B}}(t) \boldsymbol{y}(t) \\
u(t) & =\boldsymbol{F}(t) \hat{\boldsymbol{x}}_{z}(t)
\end{aligned}
$$

where the details of the matrices and output $u(t)$ is constructed by

$$
\begin{aligned}
\hat{\boldsymbol{A}}(t) & =\boldsymbol{A}_{z}(t)+\boldsymbol{B}_{2}(t) \boldsymbol{F}(t)+\gamma^{-2}(t) \boldsymbol{B}_{1}(t) \boldsymbol{B}_{1}^{T}(t) \boldsymbol{X}(t)-\hat{\boldsymbol{B}}(t)\left[\boldsymbol{C}_{2}(t)+\gamma^{-2}(t) \boldsymbol{D}_{21}(t) \boldsymbol{B}_{1}^{T}(t) \boldsymbol{X}(t)\right] \\
\hat{\boldsymbol{B}}(t) & =\left[\boldsymbol{I}-\gamma^{-2}(t) \boldsymbol{Y}(t) \boldsymbol{X}(t)\right]^{-1}\left[\boldsymbol{Y}(t) \boldsymbol{C}_{2}^{T}(t)+\boldsymbol{B}_{1}(t) \boldsymbol{D}_{21}^{T}(t)\right]\left[\boldsymbol{D}_{21}(t) \boldsymbol{D}_{21}^{T}(t)\right]^{-1} \\
\boldsymbol{F}(t) & =-\tilde{\boldsymbol{R}}(t)^{-1}\left[\boldsymbol{B}_{2}^{T}(t) \boldsymbol{X}(t)+\tilde{\boldsymbol{S}}^{T}(t)\right]
\end{aligned}
$$

where $\boldsymbol{X}(t)$ and $\boldsymbol{Y}(t)$ are given by solving the following Riccati differential equations with an explicit method such as the Runge-Kutta method.

$$
\begin{aligned}
-\dot{\boldsymbol{X}}(t)= & \boldsymbol{X}(t) \boldsymbol{A}_{z}(t)+\boldsymbol{A}_{z}^{T}(t) \boldsymbol{X}(t)+\boldsymbol{X}(t) \boldsymbol{B}_{1}(t) \boldsymbol{B}_{1}^{T}(t) \boldsymbol{X}(t) / \gamma^{2}(t)-\left[\boldsymbol{X}(t) \boldsymbol{B}_{2}(t)+\tilde{\boldsymbol{S}}(t)\right] \tilde{\boldsymbol{R}}^{-1}(t) \\
& \times\left[\boldsymbol{B}_{2}^{T}(t) \boldsymbol{X}(t)+\tilde{\boldsymbol{S}}^{T}(t)\right]+\tilde{\boldsymbol{Q}}(t), \quad \boldsymbol{X}\left(t_{f}\right)=\boldsymbol{0} \\
\dot{\boldsymbol{Y}}(t)= & \boldsymbol{Y}(t) \boldsymbol{A}_{z}^{T}(t)+\boldsymbol{A}_{z}(t) Y(t)+\boldsymbol{Y}(t) \boldsymbol{C}_{1}^{T}(t) \boldsymbol{C}_{1}(t) \boldsymbol{Y}(t) / \gamma^{2}(t)-\left[\boldsymbol{Y}(t) \boldsymbol{C}_{2}^{T}(t)+\boldsymbol{B}_{1}(t) \boldsymbol{D}_{21}^{T}(t)\right] \\
\times & {\left[\boldsymbol{D}_{21}(t) \boldsymbol{D}_{21}^{T}(t)\right]^{-1}\left[\boldsymbol{C}_{2}(t) \boldsymbol{Y}(t)+\boldsymbol{D}_{21}(t) \boldsymbol{B}_{1}(t)\right]+\boldsymbol{B}_{1}(t) \boldsymbol{B}_{1}^{T}(t), \quad \boldsymbol{Y}(0)=\boldsymbol{0} }
\end{aligned}
$$

where the solutions $\boldsymbol{X}_{w}(t)$ and $\boldsymbol{X}_{u}(t)$, which are the components in the formulation of $u^{o}$ and $\boldsymbol{w}_{d}^{o}$, coincide with $\boldsymbol{X}(t) \geq 0$ derived from the above Riccati differential equation ${ }^{(12)}$.

For the nonstationary robust control method, the design function $\gamma(t)$ on the worst disturbance is constructed in such a manner that depends on the variation of uncertainty. For instance, by letting $\gamma(t) \rightarrow \infty$ or $\gamma(t)$ have large values the $\mathrm{H}^{2}$ optimal control is performed ${ }^{(7)}$. In this paper, for implementing the continuous $\mathrm{H}^{\infty}$ quasi-optimal control, it is focused to assign the smallest possible value to $\gamma(t)$ at the arbitrary time. The parameter $\gamma(t)$ is designed on the basis of $\gamma\left(t_{i}\right)$ which is derived from the values given by the iteration of the $\gamma$-iteration at the arbitrary time $t_{i}$. However, the $\gamma(t)$ derived from the iteration cannot be utilized directly to design a robust controller because the Riccati differential equations (24) and (25) have no stable solutions based on it. Resultingly, by employing the larger $\gamma(t)$ referring the $\gamma\left(t_{i}\right)$, the quasi optimal controller is obtained. 


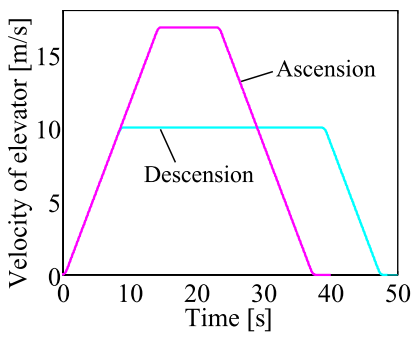

Fig. 3 Time history of velocity of the elevator

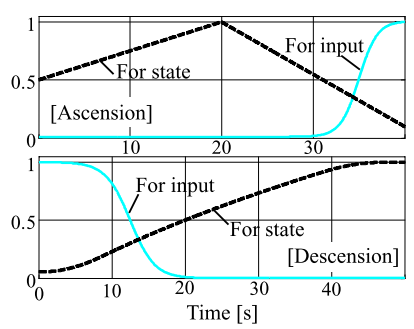

Fig. 6 Shape of the time-varying weightings

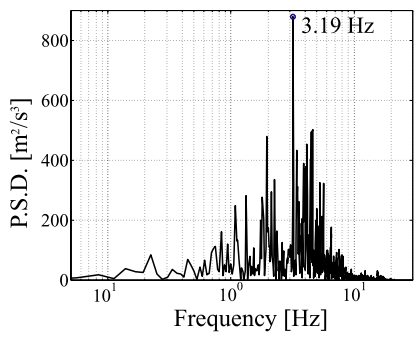

Fig. 4 Power spectrum density of the acceleration of the Chi-Chi earthquake

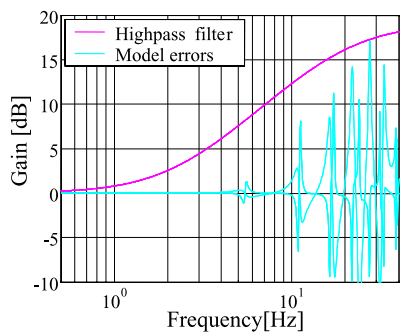

Fig. 7 Shape of the highpass filter enveloping model errors

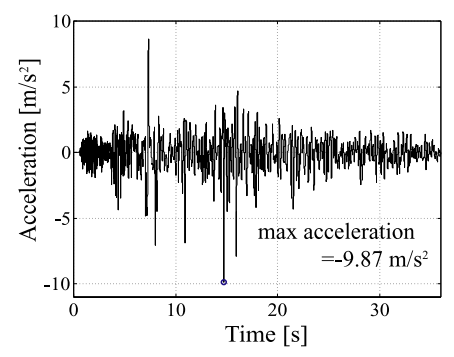

Fig. 5 Time history of the acceleration of the Chi-Chi earthquake

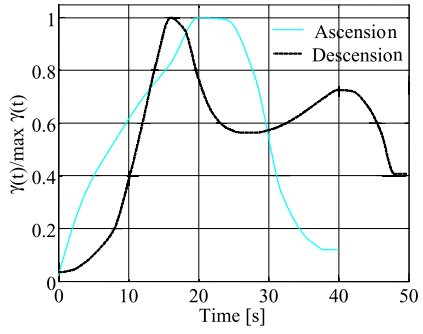

Fig. 8 Shape of the sensitivity parameter $\gamma(\mathrm{t})$

\section{Numerical calculation}

The performance and robustness against the tension change are examined through the numerical calculation based on the model and method in the previous sections.

\subsection{Condition of the numerical calculation}

First, in the numerical calculation, they are determined as the height of the building is $500 \mathrm{~m}$; the mass of the building $5.0 \times 10^{8} \mathrm{~kg}$; the first natural frequency of the building 0.11 $\mathrm{Hz}$; the damping ratio of all the mode of the building $1.0 \%$; the mass of the traction sheave $2.0 \times 10^{4} \mathrm{~kg}$; the line density of the rope $1.7 \mathrm{~kg} / \mathrm{m}$; the damping ratio of the rope $5.0 \%$; and the mass of the cage $7.5 \times 10^{3} \mathrm{~kg}$. Further, the maximum speed of the elevator is $1000 \mathrm{~m} / \mathrm{min}$ during its ascending motion and $600 \mathrm{~m} / \mathrm{min}$ during its descending motion as shown in Fig3. In this case, the distance for which the elevator runs is $400 \mathrm{~m}$ and the term, when the building is subjected to the earthquake, is 40 seconds during the ascending motion or 50 seconds during the descending motion. The earthquake occurs from the start of the elevator to the above end time. Furthermore, for the control device, a $0.75 \mathrm{~kW}$ alternate current motor is employed as an actuator and the parameters of the control device are then assigned as $\alpha_{a}=4.4 \times 10^{5}$, $\beta_{a}=1.2 \times 10^{2}, \gamma_{a}=5.9 \times 10^{2}, \epsilon_{a}=2.6 \times 10^{-3}$, the maximum voltage into the motor is 200 $\mathrm{V}$ and the maximum current into the motor is $13 \mathrm{~A}$, The parameters of the gap are determined in a manner that $k_{a}=1.0 \times 10^{7} \mathrm{~kg} / \mathrm{s}^{2}, c_{a}=7.5 \times 10^{3} \mathrm{~kg} / \mathrm{s}$ and the nominal gap-width equals $1.0 \times 10^{-2} \mathrm{~m}$. In addition to the previous conditions, the discrete number of the rope is 10 for designing the controller and 20 for the verification of the controller performance; namely, the model error of the controlled object is expressed by the difference of the discrete numbers in the models. Moreover, the fourth order Runge-Kutta method is adopted as the integrating method of the differential equation and the interval of the integrating time is then set $0.5 \mathrm{~ms}$.

Second, the two controllers, which are employed in the numerical calculation, are designed on the basis of the nonstationary optimal control method (Optimal) and the nonstationary robust control method considering the uncertainties (Robust), respectively. For the practical verification, it is assumed that the five observable states are the relative displacement and velocity of the traction sheave from the building, the relative displacement of the control device from the building, and the relative displacement and velocity of the cage of the elevator from the building. Further, the position where the control device is located is the bottom of the 
second discrete element of the rope and the lengths of the first and second elements of the rope are then fixed. The simple disturbance which has the power peaks in the vicinity of the first and second natural frequency of the building, namely 0.1 and $0.3 \mathrm{~Hz}$, and the 9.21 Chi-Chi earthquake in 1999 in Taipei are adopted as the disturbances for the design of the controller and verification of the performance of the controller, respectively. The power spectrum density and time history of the acceleration of the Chi-Chi earthquake are shown in Figs. 4 and 5.

Finally, in the numerical calculation, the robustness against the variation of the rope tension due to fluctuation of quality of the rope and incoming and outgoing by passenger is considered. For the control of the single rope, the verification of the robustness of both controllers is performed for the case in which the building is subjected to the simple disturbance and tension of the rope is then varied in the range from $\pm 0 \%$ to $\pm 20 \%$. The performance of both controllers for the simultaneous control of the multiple ropes is examined for the case in which the building is subjected to the Chi-Chi earthquake and tension of the rope is then varied in the range from $\pm 0 \%$ to $\pm 10 \%$. For the comparison of the performances of the controllers, their gains are adjusted such that the force of one controller is equal to another force with regard to its rms value in the time domain for the case in which the building is subjected to the simple disturbance. The amplitude of the simple disturbance is also adjusted as the absolute maximum displacement of the rope is approximately less than 0.5 meters in the case with no control; meanwhile, the absolute maximum acceleration of the Chi-Chi earthquake is regulated within $5.0 \mathrm{~m} / \mathrm{s}^{2}$.

\subsection{Synthesis of the controller}

For designing the controller, there are some weightings to be shaped: the time-varying weightings on the state of the controlled object, input, observation noise and system noise; particularly in the design of the robust controller, the weightings on the structured and unstructured uncertainties in time and frequency domain, respectively, and the sensitivity parameter $\gamma(t)$. We can enable all of the above weightings to be time-varying. First, the time-varying weighting on the control input is expressed by using the smooth sigmoid function so that the controller suppress the vibration of the rope when its length is short and it is shown in Fig6. The weighting on the state of the controlled object is designed as shown in Fig6 because the displacement of the rope has the maximum value at 20 seconds during ascension or in the vicinity of the end time during descension.

Second, for designing the robust controller considering the unstructured uncertainty, the modeling error as the unstructured uncertainty comes of the ignored high order modes of the controlled object; in this paper, they are expressed by the differences in the frequency responses under control and non-control which are the responses from the single control input $u$ to all the observed outputs $\boldsymbol{y}$. Hence, the order of the rope model as all the modes are under control is set 40 and the order of the lower model is set 10. The first order high-pass filter is applied to note the unstructured uncertainty and it is then designed to envelop all the modeling errors in the frequency domain as shown in Fig7. The quantity of the structured uncertainty due to the parameter variation is estimated with the differences between the parameter-varying and parameter-invariant models in the time domain. The weighting on the structured uncertainty is then derived from the absolute maximum differences between their system matrices through the entire time. Further, the parameter-invariant row and column in the matrices are removed so that the dimension of the weighting on the structured uncertainty becomes lower. The derived weighting is embedded in the generalized plant; however, the input, disturbance and output matrices are not weighted because their parameters are not varied. Finally, the normalized $\gamma(t)$ which is shaped on the basis of the minimum $\gamma$ derived from the discrete $\gamma$ iteration is shown in Fig8 and it is then confirmed that the $\gamma(t)$ is influenced by the other time-varying weightings. 
Table 1 Nominal control results (Sine-Wave case)

\begin{tabular}{|c|c|c|c|c|c|c|c|}
\hline \multicolumn{2}{|c|}{ Sine wave } & Max x & r.m.s x & Max u & r.m.s. u & Max stroke & Max i \\
\hline \multirow{4}{*}{ Up } & Non-control & 0.492 & 0.107 & - & - & - & - \\
\cline { 2 - 9 } & Optimal & 0.346 & 0.0718 & 97.9 & 31.5 & 0.145 & 5.68 \\
\cline { 2 - 8 } & Robust & 0.259 & 0.0616 & 71.9 & 31.5 & 0.202 & 4.42 \\
\hline \multirow{4}{*}{ Down } & Non-control & 0.477 & 0.100 & - & - & - & - \\
\cline { 2 - 8 } & Optimal & 0.235 & 0.0553 & 70.0 & 22.9 & 0.126 & 1.75 \\
\cline { 2 - 8 } & Robust & 0.205 & 0.0510 & 67.0 & 22.8 & 0.105 & 1.73 \\
\hline
\end{tabular}

Table 2 Control results (Chi-Chi earthquake case)

\begin{tabular}{|c|c|c|c|c|c|c|c|}
\hline \multicolumn{2}{|c|}{ Chi-Chi earthquake } & $\operatorname{Max} x$ & r.m.s x & Max u & r.m.s. u & Max stroke & $\operatorname{Max} \mathrm{i}$ \\
\hline \multirow[t]{4}{*}{ Up } & Non-control & 0.749 & 0.182 & - & - & - & - \\
\hline & Optimal & 0.597 & 0.114 & 200 & 54.9 & 0.302 & 9.10 \\
\hline & Robust & 0.459 & 0.0885 & 200 & 71.8 & 0.469 & 8.18 \\
\hline & Three ropes & 0.446 & 0.0930 & 200 & 97.9 & 0.414 & 12.6 \\
\hline \multirow[t]{4}{*}{ Down } & Non-control & 0.546 & 0.148 & - & - & - & - \\
\hline & Optimal & 0.297 & 0.0919 & 153 & 51.4 & 0.204 & 3.94 \\
\hline & Robust & 0.311 & 0.0850 & 171 & 58.3 & 0.252 & 4.53 \\
\hline & Three ropes & 0.308 & 0.0841 & 196 & 71.8 & 0.275 & 5.56 \\
\hline
\end{tabular}

\subsection{Result of the numerical calculation for the case to control the vibration of a single} rope and consideration of the result

In this control problem, from the viewpoint of the damage to the elevator system, it is focused that the maximum displacement of the rope is reduced. In addition, it is expected to extend the life cycle of the rope by reducing the maximum displacement of the rope in comparison with the case in which the root mean square value of the displacement is reduced.

Tables 1 and 2 show the result of the numerical calculation that simulates the vibration control of the single rope in the nominal case in which the tension of the rope is not varied and the building is subjected to the simple disturbance or the Chi-Chi earthquake. In these tables, 'Max' means the absolute maximum value; 'r.m.s' the root mean square value; ' $x$ ' the entire displacement of the rope; ' $u$ ' the input voltage into the motor; 'stroke' the displacement of the motor; and 'i' the current into the motor. They are also shown in Figs. 9-12; the time histories of the displacement at the center of the rope and absolute maximum displacement of the rope through the entire control period for the case in which the tension of the rope is not varied during ascension, the reduction rate of the absolute maximum displacement of the rope for the case in which the tension of the rope is varied in the range from 0.8 to 1.2 times of the nominal value and the time history of the displacement of the rope at the position where the control device is located. Furthermore, the result during descension is shown in a similar way in Figs. 10, 11, 13 and 14. The results in Tables 1 and 2 indicate that all the controllers have the approximately equivalent performances only for the nominal case. The effective suppression for the vibration of the rope is found at 20 seconds when the absolute maximum displacement of the rope occurs in the case without control as shown in Fig9. It is also confirmed in Fig12 that the dynamics of the rope are valid for the simulation of the contact between the rope and control device. With regard to the performance of the controller during ascension, the nonstationary robust controller shows the efficient vibration suppression using the identical control power with the power calculated by the nonstationary optimal controller. The reason why the nonstationary optimal controller cannot obtain the good performance for the vibration suppression is that the control gain is smaller than the robust one. However, to increase the gain in the optimal controller is not implemented because the current of the motor is more increased for the case in which the building is subjected to the Chi-Chi earthquake. As the result, the nonstationary robust controller requires a smaller capacity motor for implementing the effective vibration suppression. During the descending motion of the elevator, the robust control shows the better robustness than the another control in terms of the maximum displacement of the rope as shown in Table 1 and Fig13. However, it is found in Table 2 that the performance of the robust controller is getting worse in terms of the reduction of the maximum displacement of the rope in the case of the Chi-Chi earthquake. This is 


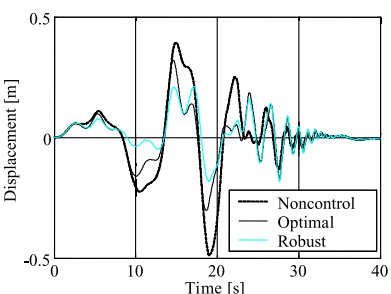

Fig. 9 Time history of the displacement at the center of rope

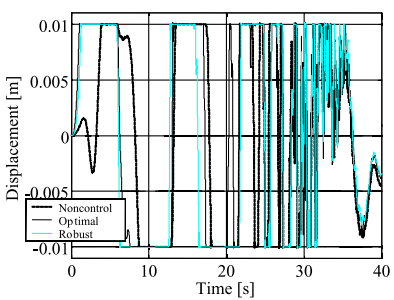

Fig. 12 Time history of the relative displacement of rope at position where the control device is located
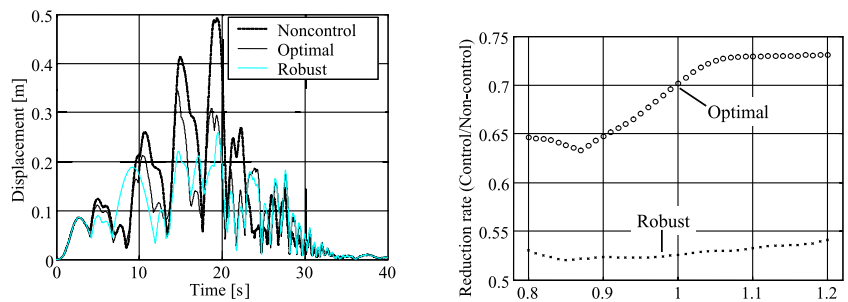

Fig. 10 Time history of the ab- Fig. 11 Reduction rate of the maxsolute maximum displacement

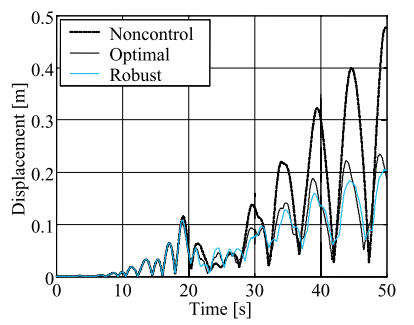

Fig. 13 Time history of the absolute maximum displacement imum displacement of rope (Control/Non-control)

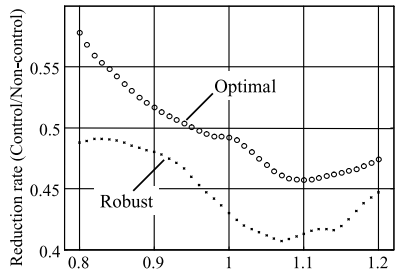

Fig. 14 Reduction rate of the maximum displacement of rope (Control/Non-control)

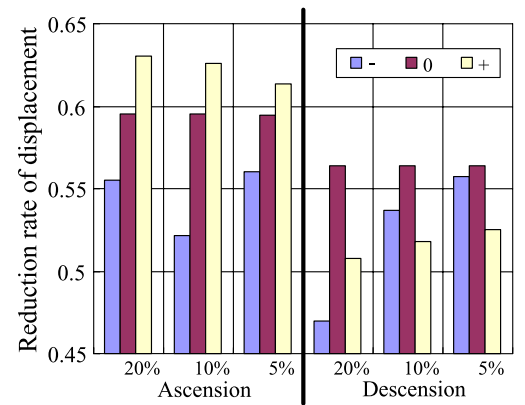

Fig. 15 Reduction rate of the maximum displacement of rope (Control/Non-control)

caused by the large disturbance added into the building while the length of the rope is short and the conservative synthesis of the robust controller at the same term. This issue is solved by the improvement of the time-varying weightings; however, the redesign of the controller depending on an earthquake is inefficient and this is inconsiderable because the rms value of the displacement of the rope is reduced when the length of the rope is long. Finally, during both of ascension and descension, the usefulness of the nonstationary robust controller against the parameter variation and modeling error is proved in Figs. 11 and 14.

\subsection{Verification of the simultaneous control for vibration of the multiple elevator ropes}

Although it is considered from the previous results that the robustness and performance in terms of the vibration control of the single rope is good, the cage is actually hung by using the ten and several ropes; hence, the multiple ropes should be simultaneously controlled using some control devices and sensors. However, it is better that the number of the actuator and sensor is few from the viewpoint of their cost and location. Hence, the feasibility of the simultaneous control of the multiple ropes using the single control device is considered in this section. In this case, the tension of the rope is different from the others. In Table 2, the result of the vibration control for the rope with the nominal tension is shown when the three ropes including it are controlled simultaneously. Figure 15 shows the reduction rate of the maximum displacement of the rope during descension and ascension for the case in which the tensions of the three ropes are set $\pm 20 \%, \pm 10 \%$ and $\pm 5 \%$ in addition to the nominal tension. The controller is designed under the assumption that all the ropes are trussed and the verified model is formulated on the assumption that each rope does not contact the other ropes. The 
effective vibration suppression of the multiple ropes and robustness against the uncertainties are derived from the above results. However, the reduction performance is getting worse than the performance in the control of the single rope. More effective performance of the vibration reduction may not be expected because of the limit of the motor performance. In particular, the input voltage and current into the motor is considerably increased during the ascending motion of the elevator. Therefore, an appropriate actuator should be considered in terms of the vibration performance and cost before the robustness of the controller in this case is discussed. Moreover, it is confirmed in the another simulation that the $50-60 \%$ vibration suppression of the ten ropes can be implemented only during the descension.

\section{Conclusions}

This paper presented a solution for the transverse vibration problem of the high-speed elevator rope based on the nonstationary robust control using the control device which moves the rope directly. The nonlinearity and dynamics of the control device due to the presence of the gaps are also verified through the numerical calculation. In the proposed method, the nonstationary robust controller was designed with considering the uncertainties due to modeling errors and parameter variation. The numerical calculation that simulates the vibration control for the single rope or three ropes by the proposed control device and method was performed; consequently, the followings were confirmed: (1) the high robustness of the nonstationary robust controller through the vibration control of the single rope in the presence of the tension variation and modeling error; (2) the adequate capability of the control using the control device with gaps for simultaneously controlling the vibration of the multiple ropes with the robustness against the tension fluctuation among the ropes.

\section{References}

( 1 ) Y. Terumichi, M. Yoshizawa, "Lateral oscillation of a moving elevator rope", Trans. JSME, series-C, Vol. 58, No. 545, (1992), pp. 17-24. (in Japanese)

( 2 ) H. Kimura, et al., "Vibration analysis and suppression of elevator rope for high-rise building", Proc. 3rd World Conf. on Struct. Cont., Vol. 3, (2004), pp. 827-832.

( 3 ) D. J. N. Limebeer, et al., "A game theoretic approach to $\mathrm{H}^{\infty}$ control for time-varying systems", SIAM J. Control and Optimization, Vol. 30, No. 2, (1992), pp. 262-283.

( 4 ) K. Takagi, H. Nishimura, "Gain-scheduled control of a tower crane considering varying load-rope length", JSME Int. J. Series-C, Vol. 42, No. 4, (1999), pp. 914-921.

( 5 ) S. Hara, K. Yoshida, "Simultaneous optimization of positioning and vibration control using time-varying criterion function", Trans. JSME, series-C, Vol. 60, No. 570, (1994), pp. 424-429. (in Japanese)

( 6 ) M. Otsuki, et al., "Vibration control for rope-sway of elevator of high-rise building", Trans. JSME, series-C, Vol. 68, No. 676, (2002), pp. 3589-3596. (in Japanese)

( 7 ) S. Hara, K. Yoshida, "A method of nonstationary robust control and its application to positioning problem of flexible structures", J. SICE, Vol. 31, No. 8, (1995), pp. 11361143. (in Japanese)

( 8 ) M. Otsuki, et al., "Experimental study on vibration control for rope-sway of elevator of high-rise building”, Proc. 2002 American Cont. Conf., (2002), pp. 238-243.

( 9 ) M. Otsuki, et al., "Nonstationary robust vibration control considering scaled structured and unstructured uncertainties", Trans. JSME, series-C, Vol. 69, No. 686, (2003), pp. 2564-2571. (in Japanese)

(10) A. Nagamatsu, et al., "Dynamics handbook", Asakura Publishing Co., Ltd., (1993), pp. 293-294. (in Japanese)

(11) J. C. Doyle, et al., "State-space solutions to standard $\mathrm{H}^{2}$ and $\mathrm{H}^{\infty}$ control problem", IEEE Trans. Auto. Cont., Vol. 34, No. 8, (1989), pp. 831-846.

(12) H. Kano, "Optimal control problems -calculus of variations, $\mathrm{H}^{\infty}$ control and viscosity solutions", J. SICE, Vol. 36, No. 11, (1997), pp. 768-775. (in Japanese) 